\title{
Prospective Comparison of the AIMS65 Score, Glasgow-Blatchford Score, and Rockall Score for Predicting Clinical Outcomes in Patients with Variceal and Nonvariceal Upper Gastrointestinal Bleeding
}

\author{
Arunchai Chang', Chokethawee Ouejiaraphant ${ }^{2}$, Keerati Akarapatima', Attapon Rattanasupa ${ }^{1}$ and Varayu Prachayakul ${ }^{3}$ \\ ${ }^{1}$ Division of Gastroenterology, Department of Internal Medicine, Hatyai Hospital, Songkhla, ${ }^{2}$ Department of Internal Medicine, Hatyai \\ Hospital, Songkhla, ${ }^{3}$ Siriraj Gastrointestinal Endoscopy Center, Division of Gastroenterology, Department of Internal Medicine, Siriraj \\ Hospital, Faculty of Medicine, Mahidol University, Bangkok, Thailand
}

Background/Aims: This study aimed to determine the performance of the AIMS65 score (AIMS65), Glasgow-Blatchford score (GBS), and Rockall score (RS) in predicting outcomes in patients with upper gastrointestinal bleeding (UGIB), and to compare the results between patients with nonvariceal UGIB (NVUGIB) and those with variceal UGIB (VUGIB).

Methods: We conducted a prospective observational study between March 2016 and December 2017. Receiver operating characteristic curve analysis was performed for all outcomes for comparison. The associations of all three scores with mortality were evaluated using multivariate logistic regression analysis.

Results: Of the total of 337 patients with UGIB, 267 patients (79.2\%) had NVUGIB. AIMS65 was significantly associated (odds ratio [OR], 1.735; 95\% confidence interval [CI], 1.148-2.620), RS was marginally associated (OR, 1.225; 95\% CI, 0.973-1.543), but GBS was not associated (OR, 1.017; 95\% CI, 0.890-1.163) with mortality risk in patients with UGIB. However, all three scores accurately predicted all other outcomes (all $p<0.05)$ except rebleeding $(p>0.05)$. Only AIMS65 precisely predicted mortality, the need for blood transfusion and the composite endpoint (all $p<0.05$ ) in patients with VUGIB.

Conclusions: AIMS65 is superior to GBS and RS in predicting mortality in patients with UGIB, and also precisely predicts the need for blood transfusion and the composite endpoint in patients with VUGIB. No scoring system could satisfactorily predict rebleeding in all patients with UGIB. Clin Endosc 2021;54:211-221

Key Words: AIMS65 score; Glasgow-Blatchford score; Outcome; Rockall score; Upper gastrointestinal bleeding

\section{INTRODUCTION}

Upper gastrointestinal bleeding (UGIB) is a common cause of hospital admission worldwide, and has a mortality rate of

\footnotetext{
Received: March 4, 2020 Revised: April 27, 2020

Accepted: May 7, 2020

Correspondence: Varayu Prachayakul

Siriraj Gastrointestinal Endoscopy Center, Division of Gastroenterology, Department of Internal Medicine, Siriraj Hospital, Faculty of Medicine, Mahidol University, 2 Tanon Wang Lang, Siriraj, Bangkoknoi, Bangkok 10700, Thailand Tel: +66-8-186-54646, Fax: +66-2-411-5013, E-mail: kaiyjr@gmail.com ORCID: https://orcid.org/0000-0001-6652-9044

(c) This is an Open Access article distributed under the terms of the Creative Commons Attribution Non-Commercial License (http://creativecommons.org/ licenses/by-nc/3.0) which permits unrestricted non-commercial use, distribution, and reproduction in any medium, provided the original work is properly cited.
}

between $2 \%$ and $15 \% .{ }^{1}$ Recent guidelines have recommended stratifying patients with UGIB into higher and lower risk categories for treatment decisions and prognostication. ${ }^{1-3}$ The widely used scoring systems include the Glasgow-Blatchford score (GBS), Rockall score (RS), and AIMS65 score (AIMS65) (Table 1); however, their role in clinical practice remains uncertain. ${ }^{4-6}$ Compared with other existing scores, AIMS65 is simple, easy to remember, can be calculated with nonweighted elements, and can be routinely evaluated in the emergency department. ${ }^{7}$

These scores have been validated and compared, in terms of their accuracy in predicting various outcomes among patients presenting with UGIB, in numerous studies. ${ }^{711}$ Most of the previous studies included both patients with nonvariceal 
Table 1. Scoring Systems

\begin{tabular}{|c|c|c|c|}
\hline Scoring system & Admission clinical factor & Parameter & Score \\
\hline \multirow[t]{5}{*}{ AIMS65 score } & Albumin & $<3.0 \mathrm{mg} / \mathrm{dL}$ & 1 \\
\hline & INR & $>1.5$ & 1 \\
\hline & Mental status & Altered & 1 \\
\hline & SBP, mm Hg & $\leq 90$ & 1 \\
\hline & Age, yr & $\geq 65$ & 1 \\
\hline \multirow[t]{13}{*}{ Rockall score } & Age, yr & $<60$ & 0 \\
\hline & & $60-79$ & 1 \\
\hline & & $\geq 80$ & 2 \\
\hline & Shock & Heart rate $>100 \mathrm{bpm}$ & 1 \\
\hline & & $\mathrm{SBP}<100 \mathrm{~mm} \mathrm{Hg}$ & 2 \\
\hline & Comorbidity & No major & 0 \\
\hline & & CHF, IHD, or major comorbidity & 2 \\
\hline & & Renal failure, liver failure, metastatic malignancy & 3 \\
\hline & Endoscopic finding & Mallory-Weiss tear or no lesion and no stigmata & 0 \\
\hline & & All other diagnoses & 1 \\
\hline & & GI malignancy & 2 \\
\hline & Stigmata of recent bleeding & No stigmata or pigmented spot on ulcer & 0 \\
\hline & & Blood in upper GI tract, adherent clot, visible vessel, bleeding & 2 \\
\hline \multirow[t]{17}{*}{ Glasgow-Blatchford score } & $\mathrm{BUN}, \mathrm{mg} / \mathrm{dL}$ & $\geq 18.2$ to $<22.4$ & 2 \\
\hline & & $\geq 22.4$ to $<28$ & 3 \\
\hline & & $\geq 28$ to $<70$ & 4 \\
\hline & & $\geq 70$ & 6 \\
\hline & Hemoglobin level, g/dL & Male $\geq 12.0$ to $<13.0$ & 1 \\
\hline & & $\geq 10.0$ to $<12.0$ & 3 \\
\hline & & $<10.0$ & 6 \\
\hline & & Female $\geq 10.0$ to $<12.0$ & 1 \\
\hline & & $<10.0$ & 6 \\
\hline & SBP, mm Hg & $\geq 100$ to $<109$ & 1 \\
\hline & & $\geq 90$ to $<100$ & 2 \\
\hline & & $<90$ & 3 \\
\hline & Other markers & Heart rate $>100 \mathrm{bpm}$ & 1 \\
\hline & & Presented with melena & 1 \\
\hline & & Presented with syncope & 2 \\
\hline & & Hepatic disease & 2 \\
\hline & & Cardiac failure & 2 \\
\hline
\end{tabular}

BUN, blood urea nitrogen; CHF, congestive heart failure; GI, gastrointestinal; IHD, ischemic heart disease; INR, international normalized ratio; SBP, systolic blood pressure. 
UGIB (NVUGIB) and patients with variceal UGIB (VUGIB), whereas some studies excluded patients with VUGIB..$^{12-14}$ Many differences exist between patients with NVUGIB and those with VUGIB, including management and natural history, resulting in different performances in the prediction of clinical endpoints between the two patient groups. Limited data are available on the efficacy of AIMS65, RS, and GBS in predicting outcomes in patients with NVUGIB compared with patients with VUGIB.

The purpose of the present study was to compare the performance of AIMS65, RS, and GBS in patients presenting with UGIB, including those with any cause of UGIB (overall UGIB [OUGIB]) and subgroups of patients with NVUGIB and VUGIB.

\section{MATERIALS AND METHODS}

\section{Study design and population}

This was a prospective observational study conducted at Hatyai Hospital (regional referral center in southern Thailand) between March 2016 and December 2017. Patients were included if they were $>18$ years old and presented to the hospital with evidence of UGIB, defined as hematemesis, coffee-ground vomiting, melena, or hematochezia with vital sign instability. Patients were excluded if they (1) had a history of UGIB in the previous 3 months, (2) had a final diagnosis of non-UGIB after the diagnostic workup, (3) had undergone endoscopy at another institution before admission, (4) had incomplete data required for the calculation of either of the three scores, and (5) refused undergoing endoscopic examination or providing informed consent for the study.

According to the protocol of the study hospital, all patients with UGIB visiting the emergency or outpatient department were initially assessed and hemodynamically stabilized. All patients were treated as inpatient cases and subsequently underwent endoscopy during the study period. The clinical management, including the time of endoscopy and use of proton pump inhibitors (PPIs) before endoscopy, was judged by each gastroenterologist depending on the patient's clinical status. In addition, for patients who had a risk for variceal bleeding, intravenous vasopressors and antibiotics were administered, and discontinued if variceal bleeding was excluded. During endoscopy, the presence of high-risk stigmata was considered an indication for endoscopic hemostasis. At our institution, the endoscopic practice for patients with high-risk stigmata of nonvariceal bleeding was injection therapy with diluted adrenaline around the culprit lesion, combined with thermal contact or mechanical clips. There was no policy of adrenaline application alone. In cases of esophageal or gastric variceal bleeding, band ligation or injection of cyanoacrylate was performed, respectively. ${ }^{15,16}$ Transjugular intrahepatic portosystemic stents were not available at our center. Postendoscopic high-dose PPIs administered via intravenous bolus followed by continuous infusion were given in cases of highrisk stigmata of nonvariceal bleeding or other selected cases considered by each individual endoscopist depending on clinical judgment. ${ }^{1-3}$ Red blood cell transfusion was considered at a hemoglobin threshold of 7-8 g/dL or when there were signs of hemodynamic instability. ${ }^{16,17}$ Consultation for surgery or embolization was considered for patients who failed endoscopic intervention or developed rebleeding despite two adequate endoscopic intervention attemps. ${ }^{1}$

\section{Data collection}

For each patient, the following data were collected: age, sex, clinical presentation, comorbidities, current medications, and laboratory results on admission. In addition, the time to endoscopy, endoscopic findings, type of endoscopic intervention, number of packed red blood cell units received, surgical and radiologic intervention, and subsequent clinical outcomes (including rebleeding and in-hospital death) were assessed.

\section{Outcomes and definition}

The primary outcome was in-hospital mortality. The secondary outcomes were (1) the need for blood transfusion; (2) endoscopic intervention requirement; (3) in-hospital rebleeding; and (4) the composite endpoint of in-hospital mortality, need for blood transfusion, overall interventions (including endoscopic, radiologic, and surgical interventions), and rebleeding. The study protocol was reviewed and approved by the Institutional Review Board of Hatyai Hospital (protocol number 80/2015), and this trial was registered in the Thai Clinical Trials Registry (number: TCTR20190222006).

The patients were considered to have altered mental status if the Glasgow Coma Scale score was $<14$, or if they were defined as experiencing "disorientation", "lethargy", "stupor", or "coma". High-risk stigmata of nonvariceal bleeding was characterized as adherent clots or nonbleeding or bleeding visible vessels. Rebleeding was defined as an objective evidence of a new episode of UGIB after the initial bleeding episode was controlled or spontaneously resolved, which was associated with hemodynamic instability or a $>2 \mathrm{~g} / \mathrm{dL}$ decrease in hemoglobin level.

\section{Statistical analysis}

Categorical variables are summarized using frequency statistics (e.g., frequencies, percentage), and were compared 
between groups using Pearson chi-square test or Fisher's exact test. Descriptive statistics (e.g., mean, standard deviation, median and range) are used for continuous variables, which were compared using Student's $t$-test and Wilcoxon rank-sum test. We examined the relationship between outcome events and each score separately, using logistic regression analysis. After univariate analysis was performed, age, sex, and other variables with $p$-values $<0.1$ were included in the multivariate regression model. Next, receiver operating characteristic (ROC) curves were constructed to assess the relationship between each score and all outcomes. The area under the ROC curves (AUROCs) were then assessed with exact binomial confidence intervals (CIs). AUROCs were tested for equality using the Delong $\chi^{2}$ test. Analyses were performed using the statistical program Stata version 15.1 (StataCorp LLC; College Station, TX, USA). A $p$-value of $<0.05$ was considered statistically significant.

\section{Study sample calculation}

The study sample size was calculated according to the studies by Hyett et al. and Bryant et al. ${ }^{18,19}$ Hyett et al. revealed that the in-hospital mortality rate was $6.5 \%$ and the AUROCs of AIMS65 and GBS for predicting in-hospital mortality were 0.93 and 0.68 , respectively. ${ }^{18}$ Bryant et al. reported that the AUROCs of RS and GBS for predicting in-hospital mortality were 0.71 and 0.72 , respectively. ${ }^{19}$ For the study sample size calculation, we predicted an in-hospital mortality rate of $6.5 \%$, AIMS65 AUROC of 0.9, GBS and RS AUROC of 0.7, and a moderate correlation between AIMS65 and the other scores $(\rho=0.4)$. To achieve a power of $90 \%$ for detecting a 0.2 difference in AUROC using a two-sided test with $\alpha=0.05$, the sample size was determined to be 310 . Assuming a $10 \%$ loss because of dropouts, the sample size was estimated to be 341 participants.

\section{RESULTS}

\section{Selected patient characteristics}

Among 352 patients, 337 who met the inclusion criteria were enrolled in the study, with 247 men and an average age of $61.1 \pm 16.5$ years. Fifteen patients were excluded because nine patients underwent endoscopy before admission, three patients had a history of UGIB in the previous 3 months, two patients were diagnosed with midgastrointestinal bleeding, and one patient had incomplete information. On the basis of endoscopy results, 267 patients (79.2\%) had NVUGIB and 70 patients $(20.8 \%)$ had VUGIB. The causes of UGIB in patients with NVUGIB were peptic ulcer disease in 153 patients (45.4\%), including 81 with gastric ulcers, 53 with duodenal ulcers, and 19 with both gastric and duodenal ulcers; gastritis/ duodenitis in 77 patients (22.8\%); Mallory-Weiss tears in 28 patients (8.3\%); reflux esophagitis in 30 patients (8.9\%); portal hypertensive gastropathy in 14 patients (4.2\%); and ulcerated tumor in 11 patients (3.3\%). The endoscopic findings of patients with VUGIB included esophageal varices in 62 (88.6\%) and gastric varices with or without esophageal varices in 8 (11.4\%). Regardless of the cause of UGIB, red blood cell transfusion and endoscopic intervention were performed in 183 patients $(54.3 \%)$ and 110 patients $(32.6 \%)$, respectively. The in-hospital mortality rate was $6.2 \%$, and bleeding recurred in 10 patients (3.0\%). Tables 2 and 3 show the demographic data and clinical outcomes of all participants and the comparisons between patients with NVUGIB and those with VUGIB, respectively. Significantly different parameters and clinical outcomes were observed between the two groups. The mean age, hypertension status, nonsteroidal anti-inflammatory drug usage, hemoglobin, platelet count, serum albumin, serum blood urea nitrogen, and serum creatinine of patients with NVUGIB were greater than those of patients with VUGIB. Conversely, patients with VUGIB were more likely to have liver disease, cancer, fresh blood via the nasogastric tube, hemodynamic instability on initial assessment, and a higher international normalized ratio (INR) than those with NVUGIB. In terms of scoring systems, patients with VUGIB had higher AIMS65 and GBS than those with NVUGIB; however, there was no significant difference in RS. The in-hospital mortality, blood transfusion requirement, and endoscopic intervention rates were significantly higher in patients with VUGIB than in those with NVUGIB. No significant difference was found in terms of rebleeding or radiologic/surgical intervention. 
Table 2. Overall Baseline Characteristics and Comparison between Patients with Nonvariceal and Variceal Upper Gastrointestinal Bleeding

\begin{tabular}{|c|c|c|c|c|}
\hline Factor & $\begin{array}{l}\text { Overall UGIB } \\
\qquad(n=337)\end{array}$ & $\begin{array}{l}\text { NVUGIB } \\
(n=267)\end{array}$ & $\begin{array}{l}\text { VUGIB } \\
(n=70)\end{array}$ & $p$-value \\
\hline Male sex & $247(73.3 \%)$ & $192(71.9 \%)$ & $55(78.6 \%)$ & 0.262 \\
\hline Age $(y r)$, mean $\pm S D$ & $61.1 \pm 16.5$ & $62.3 \pm 17.2$ & $56.1 \pm 12.7$ & 0.001 \\
\hline Body mass index $\left(\mathrm{kg} / \mathrm{m}^{2}\right)$, mean $\pm \mathrm{SD}$ & $23.2 \pm 4.6$ & $23.2 \pm 4.7$ & $23.0 \pm 4.1$ & 0.771 \\
\hline Fresh blood via nasogastric tube & $73(21.7 \%)$ & $42(15.2 \%)$ & $31(51.7 \%)$ & $<0.001$ \\
\hline Shock (hemodynamic instability) & $45(13.6 \%)$ & $29(10.9 \%)$ & $16(22.9 \%)$ & 0.009 \\
\hline Syncope & $109(32.3 \%)$ & $83(32.1 \%)$ & $26(37.1 \%)$ & 0.335 \\
\hline History of UGIB & $6(1.8 \%)$ & $5(1.9 \%)$ & $1(1.4 \%)$ & 1.000 \\
\hline Mental status change & $8(2.4 \%)$ & $6(2.2 \%)$ & $2(2.9 \%)$ & 0.673 \\
\hline \multicolumn{5}{|l|}{ Comorbidity } \\
\hline None & $175(51.9 \%)$ & $132(49.4 \%)$ & $30(42.9 \%)$ & 0.327 \\
\hline Hypertension & $106(31.5 \%)$ & $96(36.0 \%)$ & $10(14.3 \%)$ & 0.001 \\
\hline Dyslipidemia & $33(9.8 \%)$ & $29(10.9 \%)$ & $4(5.7 \%)$ & 0.197 \\
\hline Ischemic heart disease & $7(2.1 \%)$ & $7(2.6 \%)$ & $0(0.0 \%)$ & 0.352 \\
\hline Renal failure & $25(7.4 \%)$ & $22(8.2 \%)$ & $3(4.3 \%)$ & 0.261 \\
\hline Cerebrovascular disease & $23(6.8 \%)$ & $22(8.2 \%)$ & $1(1.4 \%)$ & 0.058 \\
\hline Any malignancies & $27(8 \%)$ & $14(5.2 \%)$ & $13(18.6 \%)$ & $<0.001$ \\
\hline Liver disease & $67(19.9 \%)$ & $18(6.7 \%)$ & $49(70.0 \%)$ & $<0.001$ \\
\hline Diabetes mellitus & $73(21.6 \%)$ & $58(21.7 \%)$ & $15(21.43 \%)$ & 0.530 \\
\hline Chronic obstructive pulmonary disease & $9(2.7 \%)$ & $9(3.4 \%)$ & $0(0.0 \%)$ & 0.213 \\
\hline \multicolumn{5}{|l|}{ Medication } \\
\hline Aspirin and/or clopidogrel & $22(6.5 \%)$ & $21(7.9 \%)$ & $1(1.4 \%)$ & 0.057 \\
\hline Warfarin & $7(2.1 \%)$ & $7(2.6 \%)$ & $0(0.0 \%)$ & 0.352 \\
\hline Non-steroidal anti-inflammatory drug & $37(11.0 \%)$ & $35(13.1 \%)$ & $2(2.9 \%)$ & 0.015 \\
\hline Corticosteroid & $2(0.6 \%)$ & $2(0.7 \%)$ & $0(0.0 \%)$ & 1.000 \\
\hline Proton pump inhibitor & $15(4.5 \%)$ & $12(4.5 \%)$ & $3(4.3 \%)$ & 1.000 \\
\hline \multicolumn{5}{|l|}{ Laboratory } \\
\hline Hemoglobin & $8.9 \pm 3.3$ & $9.1 \pm 3.4$ & $8.2 \pm 2.3$ & 0.007 \\
\hline Platelet count $\left(\times 10^{3} / \mu \mathrm{L}\right)$, median with IQR & $228(162,295)$ & $221(158,294)$ & $90(62,124)$ & $<0.001$ \\
\hline Albumin $(\mathrm{mg} / \mathrm{dL})$, mean $\pm \mathrm{SD}$ & $3.18 \pm 0.76$ & $3.30 \pm 0.74$ & $2.69 \pm 0.64$ & $<0.001$ \\
\hline INR, median with IQR & $1.17(1.06,1.39)$ & $1.12(1.05,1.27)$ & $1.48(1.32,1.74)$ & $<0.001$ \\
\hline BUN (mg/dL), median with IQR & $29.0(16.0,49.0)$ & $32.0(17,52)$ & $24.0(15,38)$ & 0.028 \\
\hline Serum creatinine $(\mathrm{mg} / \mathrm{dL})$, median with IQR & $1.04(0.79,1.41)$ & $1.04(0.82,1.43)$ & $0.82(0.63,1.22)$ & $<0.001$ \\
\hline Endoscopy in $24 \mathrm{hr}$ & $214(63.5 \%)$ & $174(65.2 \%)$ & $40(57.1 \%)$ & 0.214 \\
\hline AIMS65 score, median with IQR & $1(0,2)$ & $1(0,2)$ & $2(1,3)$ & $<0.001$ \\
\hline Rockall score, median with IQR & $3(2,4)$ & $3(2,4)$ & $2.5(2,4)$ & 0.214 \\
\hline Glasgow-Blatchford score, median with IQR & $10(6,13)$ & $10(5,12)$ & $11(8,13)$ & 0.009 \\
\hline
\end{tabular}

BUN, blood urea nitrogen; INR, international normalized ratio; IQR, interquartile range; NVUGIB, nonvariceal upper gastrointestinal bleeding; SD, standard deviation; UGIB, upper gastrointestinal bleeding; VUGIB, variceal upper gastrointestinal bleeding. 


\section{Accuracy of scoring systems and comparison between patients with NVUGIB and those with VUGIB}

\section{Mortality}

AIMS65, GBS and RS were accurate in predicting mortality in patients with OUGIB (AUROC; AIMS65 =0.747, GBS $=0.671$, RS $=0.669$, all $p<0.05)$ and NVUGIB (AUROC; AIMS65 $=0.706, \mathrm{GBS}=0.699, \mathrm{RS}=0.750$, all $p<0.05$ ). When comparing the AUROCs between the scoring systems, the three scores resulted in similar mortality predictions in both patients with OUGIB and patients with NVUGIB (all $p>0.05)$. In contrast to the finding on patients with NVUGIB, only AIMS65 could precisely predict mortality in patients with VUGIB (AUROC; AIMS65 $=0.741, p=0.020$ vs. $\mathrm{GBS}=0.589$, $p=0.390$ vs. $\mathrm{RS}=0.611, p=0.284)$ (Fig. 1 ).

\section{Need for blood transfusion}

All studied scores could predict the need for blood transfusion in patients with OUGIB (AUROC; AIMS65 =0.693, $\mathrm{GBS}=0.766$, RS $=0.624$, all $p<0.001)$ and NVUGIB $(\mathrm{AU}-$ ROC; AIMS65 $=0.693$, GBS $=0.789, \mathrm{RS}=0.659$, all $p<0.001)$ (Fig. 2A). In patients with OUGIB, the AUROC for GBS and AIMS65 revealed similar accuracies (AUROC; GBS $=0.766$ vs. AIMS65 $=0.693, p=0.766)$, which were significantly higher than that associated with RS $(\mathrm{AUROC}=0.624$, all $p<0.05)$. However, in patients with NVUGIB, the AUROC of GBS for predicting the need for blood transfusion was the highest, and was significantly higher than that of either AIMS65 or RS (all $p<0.001$ ). In contrast, only AIMS65 was effective in predicting the need for blood transfusion in patients with VUGIB (AU$\mathrm{ROC}$; $\mathrm{AIMS} 65=0.658, p=0.035$ vs. $\mathrm{GBS}=0.645, p=0.052$ vs. $\mathrm{RS}=0.523, p=0.757)$.

\section{Endoscopic intervention requirement}

AIMS65, GBS, and RS revealed significant accuracy in predicting the need for endoscopic intervention in patients with OUGIB (AUROC; AIMS65 =0.619 vs. GBS =0.645 vs. RS $=0.600$, all $p<0.05$ ) and NVUGIB (AUROC; AIMS65 $=0.604$ vs. $\mathrm{GBS}=0.667$ vs. $\mathrm{RS}=0.707$, all $p<0.05)$. There was no significant difference in predicting endoscopic intervention requirements in patients with OUGIB (all $p>0.05$ ). However, among patients with NVUGIB, RS showed the highest AUROC, which was significantly higher than that of AIMS65 $(p=0.017)$ and was not significantly different from that of GBS $(p=0.307)$. However, none of the scoring systems exhibited significant accuracy in predicting the need for endoscopic intervention in the VUGIB group (AUROC; AIMS65 $=0.542$ vs. $\mathrm{GBS}=0.523$ vs. $\mathrm{RS}=0.420$, all $p>0.05$ ) (Fig. 2B).

\section{Rebleeding}

None of the scoring systems could predict rebleeding in the OUGIB group (AUROC; AIMS65 $=0.614$ vs. $\mathrm{GBS}=0.617$ vs. $R S=0.567$, all $p>0.05$ ) or in the NVUGIB (AUROC; AIMS65 $=0.657$ vs. $\mathrm{GBS}=0.682$ vs. $\mathrm{RS}=0.596$, all $p>0.05$ ) and VUGIB (AUROC; AIMS65 $=0.275$ vs. GBS $=0.094$ vs. $\mathrm{RS}=0.319$, all $p>0.05)$ subgroups (Fig. 2C).

\section{Composite endpoint}

Fig. 2D. displays the ROC and AUROCs of AIMS65, GBS, and RS for predicting the composite endpoint. All three scores showed significant predictive accuracy for predicting the composite endpoint among patients with OUGIB (AUROC; AIMS65 $=0.734$ vs. $\mathrm{GBS}=0.800$ vs. $\mathrm{RS}=0.660$, all $p<0.001)$ and NVUGIB (AUROC; AIMS65 $=0.714$ vs. GBS $=0.810$ vs. $\mathrm{RS}=0.705$, all $p<0.001)$. In patients with OUGIB, GBS
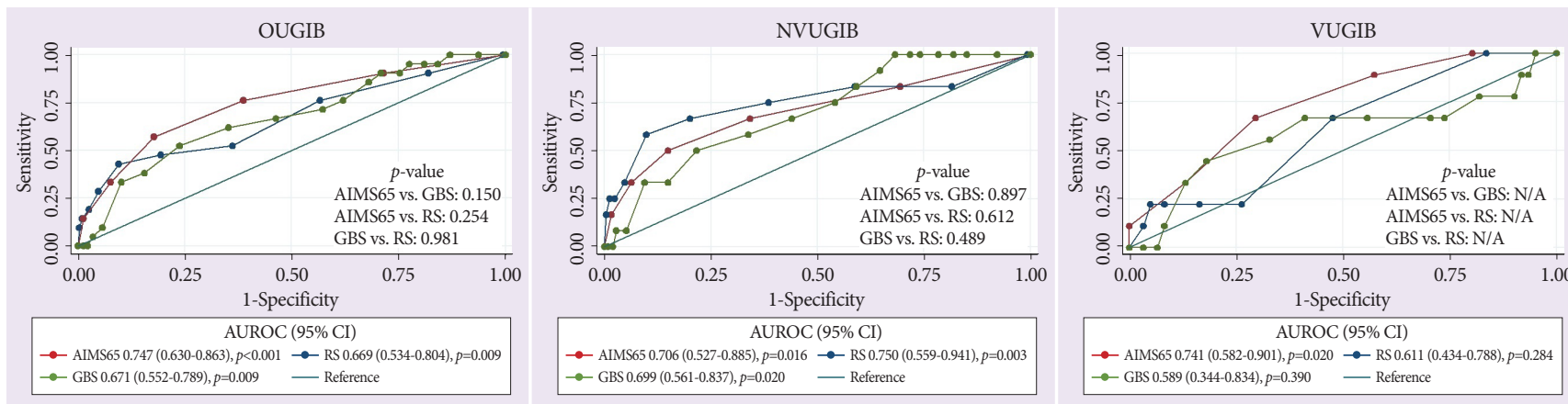

Fig. 1. A comparison of the area under the receiver operating characteristic curve (AUROC) of the AIMS65 score (AIMS65), Glasgow-Blatchford score (GBS), and Rockall score (RS) in predicting in-hospital mortality in the overall upper gastrointestinal bleeding (OUGIB) patients and patients with nonvariceal upper gastrointestinal bleeding (NVUGIB) and variceal upper gastrointestinal bleeding (VUGIB). Cl, confidence interval; N/A, not available. 

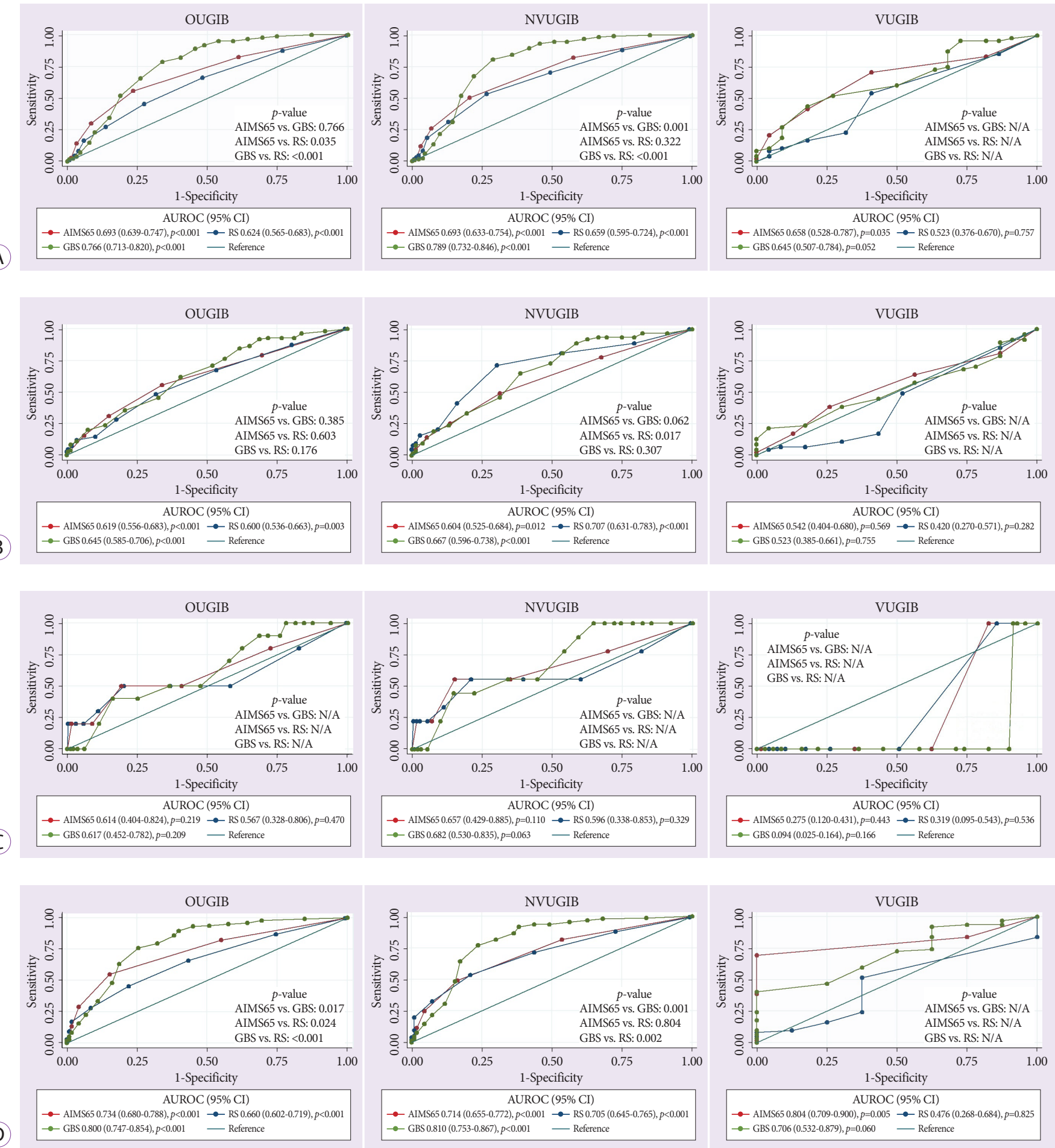

Fig. 2. A comparison of the area under the receiver operating characteristic curve (AUROC) of the AIMS65 score (AIMS65), Glasgow-Blatchford score (GBS), and Rockall score (RS) in predicting (A) the need for blood transfusion; (B) endoscopic intervention requirement; (C) rebleeding; and (D) the composite endpoint of inpatient mortality, need for blood transfusion, overall interventions, and rebleeding in the overall upper gastrointestinal bleeding (OUGIB) patients and patients with nonvariceal gastrointestinal bleeding (NVUGIB) and variceal gastrointestinal bleeding (VUGIB). Cl, confidence interval; N/A, not available. 
Table 3. Overall Clinical Outcome and Comparison between Patients with Nonvariceal and Variceal Upper Gastrointestinal Bleeding

\begin{tabular}{lcccc}
\hline Outcome & Overall UGIB $(\boldsymbol{n = 3 3 7 )}$ & NVUGIB $(\boldsymbol{n}=\mathbf{2 6 7})$ & VUGIB $(\boldsymbol{n}=\mathbf{7 0})$ & $\boldsymbol{p}$-value \\
\hline In-hospital mortality & $21(6.2 \%)$ & $12(4.5 \%)$ & $9(12.9 \%)$ & 0.022 \\
Rebleeding & $10(3.0 \%)$ & $9(3.4 \%)$ & $1(1.4 \%)$ & 0.694 \\
Blood transfusion & $183(54.3 \%)$ & $135(50.6 \%)$ & $48(68.6 \%)$ & 0.007 \\
Endoscopic intervention & $110(32.6 \%)$ & $63(23.6 \%)$ & $47(67.1 \%)$ & $<0.001$ \\
Surgical and/or radiologic intervention & $4(1.2 \%)$ & $4(1.5 \%)$ & $0(0.0 \%)$ & 0.584 \\
\hline
\end{tabular}

NVUGIB, nonvariceal upper gastrointestinal bleeding; UGIB, upper gastrointestinal bleeding; VUGIB, variceal upper gastrointestinal bleeding.

Table 4. The Association of the AIMS65 Score, Glasgow-Blatchford Score and Rockall Score with Risk of In-Hospital Mortality in Patients Regardless of the Cause of Upper Gastroesophageal Bleeding

\begin{tabular}{|c|c|c|c|c|c|c|c|c|}
\hline $\begin{array}{l}\text { Patient } \\
\text { population }\end{array}$ & Scoring system & Mortality & $n(\%)$ & $\begin{array}{c}\text { Score values } \\
\text { (median } \\
\text { with IQR) }\end{array}$ & $\begin{array}{l}\text { Unadjusted odds } \\
\text { ratio }(95 \% \mathrm{CI})\end{array}$ & $p$-value & $\begin{array}{l}\text { Adjusted odds ratio } \\
(95 \% \mathrm{CI})\end{array}$ & $p$-value \\
\hline \multirow{6}{*}{$\begin{array}{l}\text { Overall UGIB } \\
(n=337)\end{array}$} & AIMS65 score & No & $316(93.8)$ & $1(0$ to 2$)$ & 1 & & 1 & \\
\hline & & Yes & $21(6.2)$ & $3(2$ to 4$)$ & $1.990(1.444-2.740)$ & $<0.001$ & $1.735(1.148-2.620)^{\mathrm{a})}$ & 0.009 \\
\hline & Glasgow- & No & $316(93.8)$ & $10(6$ to 12$)$ & 1 & & 1 & \\
\hline & Blatchford score & Yes & $21(6.2)$ & 13 (9 to 15$)$ & $1.145(1.024-1.279)$ & 0.017 & $1.017(0.890-1.163)^{\mathrm{b})}$ & 0.801 \\
\hline & Rockall score & No & $316(93.8)$ & $3(2$ to 4$)$ & 1 & & 1 & \\
\hline & & Yes & $21(6.2)$ & $4(3$ to 7$)$ & $1.448(1.191-1.760)$ & $<0.001$ & $1.225(0.973-1.543)^{c)}$ & 0.084 \\
\hline
\end{tabular}

$\mathrm{CI}$, confidence interval; IQR, interquartile range; UGIB, upper gastrointestinal bleeding.

a) AIMS65 score: adjusted for gender, malignancy, hemoglobin, platelet count, creatinine.

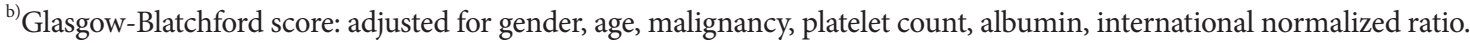

${ }^{c}$ Rockall score: adjusted for gender, malignancy, hemoglobin, platelet count, albumin, international normalized ratio, creatinine.

Table 5. Comparison of AIMS65 Score, Glasgow-Blatchford Score and Rockall Score with Estimated Optimal Cutoff Values for In-Hospital Death

\begin{tabular}{|c|c|c|c|c|c|c|}
\hline Score & Cutoff value & Sensitivity (\%) & Specificity (\%) & $\begin{array}{c}\text { Total (sensitivity } \\
+ \text { specificity) }\end{array}$ & PPV (\%) & NPV (\%) \\
\hline \multirow[t]{3}{*}{ AIMS65 score } & 2 & 76.2 & 61.4 & 137.6 & 11.6 & 97.5 \\
\hline & 3 & 57.1 & 82.3 & 139.4 & 17.6 & 96.7 \\
\hline & 4 & 33.3 & 92.4 & 125.7 & 22.6 & 95.4 \\
\hline \multirow[t]{3}{*}{ Glasgow-Blatchford score } & 11 & 66.7 & 53.8 & 120.5 & 8.8 & 96.0 \\
\hline & 12 & 61.9 & 96.2 & 158.1 & 10.5 & 96.2 \\
\hline & 13 & 52.4 & 76.3 & 128.7 & 12.8 & 96.0 \\
\hline \multirow[t]{3}{*}{ Rockall score } & 5 & 47.6 & 80.7 & 128.3 & 14.1 & 95.9 \\
\hline & 6 & 42.9 & 90.5 & 133.4 & 23.1 & 96.0 \\
\hline & 7 & 28.6 & 95.3 & 123.9 & 28.6 & 95.3 \\
\hline
\end{tabular}

NPV, negative predictive value; PPV, positive predictive value. 
performed best in detecting the composite endpoint, demonstrating better ability than AIMS65 (AUROC; 0.800 vs. 0.734, $p=0.017$ ) and RS (AUROC; 0.800 vs. $0.660, p<0.001$ ). Furthermore, GBS was the most precise in predicting the composite endpoint, significantly outperforming both AIMS65 (AUROC; 0.810 vs. $0.714, p=0.001$ ) and RS (AUROC; 0.810 vs. $0.705, p=0.002$ ) in the subgroup of patients with NVUGIB. However, in patients with VUGIB, only AIMS65 was accurate in predicting the composite endpoint (AUROC; $\operatorname{AIMS} 65=0.804, p=0.005$ vs. $G B S=0.706, p=0.060$ vs. $\mathrm{RS}=0.476, p=0.825$ ).

\section{Association of scoring systems with mortality risk, and optimal cutoff values}

The association of the scoring systems with the risk of mortality in patients with OUGIB is summarized in Table 4. After adjustment for confounding factors, AIMS65 was positively associated with the risk of death (odds ratio [OR], 1.735; 95\% CI, 1.148-2.620), whereas RS was marginally associated with the mortality risk (OR, 1.225; 95\% CI, 0.973-1.543). No significant association was observed between GBS and the risk of death (OR, 1.017; 95\% CI, 0.890-1.163).

The cutoff values that maximized the sum of the sensitivity and specificity of each score for predicting mortality were separately calculated (Table 5). The optimal cutoffs for AIMS65, GBS, and RS were 3,12 , and 6 , respectively.

\section{DISCUSSION}

Current guidelines recommend the use of risk stratification to determine priorities and select high-risk patients who should be treated with aggressive resuscitation and appropriate intervention, in order to minimize morbidity and mortality. ${ }^{1,2}$ Previous studies supported the accuracies of AIMS65, GBS, and RS in predicting outcomes or disease-related interventions. ${ }^{7,11,20}$ The main results of the current study were as follows: first, AIMS65 was found to be precise, and was not significantly different from GBS and RS in terms of the prediction of in-hospital mortality in unselected patients with UGIB. Second, all three scores were accurate tools for predicting in-hospital mortality, the need for blood transfusion, endoscopic intervention requirement, and the composite endpoint among patients with NVUGIB but not for predicting rebleeding. GBS had the best accuracy for predicting the need for blood transfusion and the composite endpoint, whereas RS performed better in predicting the need for endoscopic intervention than AIMS65 but did not differ from GBS. In contrast to patients with NVUGIB, in patients with VUGIB, AIMS65 was the only score that was a precise risk assessment tool for predicting mortality, the need for blood transfusion, and the composite endpoint during admission. None of the studied scores could predict endoscopic intervention requirement or rebleeding in this population. Third, the in-hospital mortality risk among patients with OUGIB was positively associated with AIMS65, and was possible for RS but not for GBS.

This comparative study supports the idea that GBS is the most appropriate scoring system for both overall patients with UGIB and the subgroup of patients with NVUGIB. This finding was consistent with that of previous studies. ${ }^{8,21,22}$ However, the currently available scoring systems have been validated, including among patients with UGIB, mostly by focusing on patients with NVUGIB. The performance of these scores in predicting outcomes in patients with cirrhosis presenting with VUGIB may be limited. Studies in Western countries have reported that the most common cause of UGIB is nonvariceal bleeding (86\%-93\%). ${ }^{6,8,23}$ In Asia, however, the prevalence of variceal bleeding is higher, accounting for approximately $16 \%-40 \%$ of UGIB cases. ${ }^{13,24}$ In our study, subgroup analysis of patients with VUGIB demonstrated that only AIMS65 was precise in predicting mortality, the need of blood transfusion, and the composite endpoint. This result confirmed the finding of previous studies. Reed et al. reported that both GBS and RS have a poor ability to predict clinical outcomes in individuals with VUGIB, ${ }^{23}$ and Thanapirom et al. reported that, in contrast to the finding in patients with NVUGIB, GBS and RS were not precise in predicting outcomes in patients with VUGIB. ${ }^{25}$ There are many possible reasons for this finding. Thanapirom et al. postulated that the lack of a known history of liver disease might lower GBS and RS at the time of presentation. ${ }^{25}$ The data of this study indicate that $30 \%$ of patients in the VUGIB group did not have a previous diagnosis of cirrhosis. Second, the endoscopic finding of high-risk varices has not been described as a component of GBS or RS. Finally, the NVUGIB group included a significant proportion of patients with a low risk for adverse events. ${ }^{26}$ According to our data, patients with NVUGIB received clinical intervention and died significantly less often than patients with VUGIB. In contrast to the finding associated with NVUGIB, the expected sensitivity and specificity of scoring systems for patients with VUGIB are low in the evaluation of clinical outcomes, resulting in lower AUROCs. Furthermore, variceal bleeding is mostly associated with massive hemorrhage, and prognosis is closely correlated with the severity of liver failure. ${ }^{24}$ Serum albumin levels and INR are components of AIMS65 and reflect the baseline liver function of patients who had chronic liver disease at the time of initial assessment. ${ }^{15}$ Hence, AIMS65 showed significant accuracy in predicting outcomes in patients with VUGIB. 
Although the utility of AIMS65 in predicting in-hospital morality has been proven in previous studies, ${ }^{27,28}$ our study additionally demonstrated that AIMS65 predicts other clinical outcomes (including the need for blood transfusion and the composite endpoint). These findings may be beneficial in supporting and providing appropriate management and medical resources for patients with VUGIB.

With respect to the prediction of rebleeding, none of the studied scores showed significant predictive accuracy in overall patients with UGIB or in the subgroup of patients with NVUGIB or VUGIB. Our data, consistent with several previous studies, support the assumption that the clinical usefulness of these scoring systems in terms of this outcome is low. ${ }^{24,29,30}$ It would be more important to develop a new precise score for predicting rebleeding in the future.

Our study demonstrated that only AIMS65 and possibly RS could be used for predicting mortality in patients with UGIB, whereas GBS could not. This finding could be explained by the fact that each score was developed independently with a different study population and purpose. AIMS65 and RS were designed to predict the risk for death, whereas GBS was designed to identify low-risk patients who could be safely treated as outpatients. Nakamura et al. ${ }^{31}$ reported that AIMS65, but not GBS, was an independent prognostic factor for overall survival in both patients with UGIB and those with lower gastrointestinal bleeding. However, a recent study by $\mathrm{Gu}$ et al. compared the performance of AIMS65, GBS, and RS in 799 patients with UGIB, including $15.6 \%$ with VUGIB. ${ }^{10}$ The authors found the benefit of all three scores for assessing the risk of in-hospital death in patients with UGIB. ${ }^{10}$ Of note, there were some differences between the studies, including the mortality rate and proportion of variceal bleeding, which may explain the inconsistency in the results. We recommend testing the association of scoring systems with the risk of in-hospital mortality in terms of larger study groups and/or different settings.

Previous studies revealed the different cutoff values for identifying a high risk of death in patients with UGIB. ${ }^{7,10,11,20}$ The optimal cutoff values in our study for predicting mortality were 3, 12, and 6 for AIMS65, GBS, and RS, respectively. The cutoff value could be affected by differences in the study population, ethnicity and geographical area, regional medical resources, and the aim of the cutoff value. Gu et al. postulated that the optimal cutoff of each scoring system should be specifically chosen depending on the population to maximize the ability to predict prognosis in patients. ${ }^{10}$

Some limitations of this study should be noted. First, this study was conducted at a single center in a regional referral hospital; hence, our results cannot be applied generally. Sec- ond, this study was powered to detect the expected difference for the primary outcome and not for the secondary outcomes. Third, the decisions with respect to any clinical interventions were made based on clinical judgment by individual gastroenterologists, which might have caused variability.

In conclusion, AIMS65 is precise and not significantly different from GBS and RS in predicting in-hospital mortality in overall patients with UGIB, regardless of a variceal or nonvariceal bleeding status. In patients with NVUGIB, all three scores were precise tools for predicting mortality, the need for blood transfusion, endoscopic requirement, and the composite endpoint. In contrast to the finding in patients with NVUGIB, only AIMS65 showed significant predictive accuracy in predicting mortality, need for blood transfusion, and the composite endpoint in patients with VUGIB. However, all three scoring systems cannot satisfactorily predict the rebleeding rate in overall patients with UGIB or the subgroups of patients with NVUGIB and VUGIB.

\section{Conflicts of Interest}

The authors have no potential conflicts of interest.

Funding

None.

\section{Author Contributions}

Conceptualization: Arunchai Chang, Varayu Prachayakul

Data curation: AC, Chokethawee Ouejiaraphant, Keerati Akarapatima, Attapon Rattanasupa

Formal analysis: $\mathrm{AC}, \mathrm{CO}$

Methodology: AC, CO, KA

Project administration: AC, CO, VP

Resources: AC, CO, KA, AR

Software: AC

Supervision: VP

Writing-original draft: AC, KA

Writing-review\&editing: VP

\section{ORCID}

Arunchai Chang:

Chokethawee Ouejiaraphant:

Keerati Akarapatima:

Attapon Rattanasupa:

Varayu Prachayakul: https://orcid.org/0000-0002-0158-2685 https://orcid.org/0000-0001-7603-5267 https://orcid.org/0000-0001-5907-0001 https://orcid.org/0000-0002-9595-5808 https://orcid.org/0000-0001-6652-9044

\section{REFERENCES}

1. Barkun AN, Bardou M, Kuipers EJ, et al. International consensus recommendations on the management of patients with nonvariceal upper gastrointestinal bleeding. Ann Intern Med 2010;152:101-113.

2. Laine L, Jensen DM. Management of patients with ulcer bleeding. Am J Gastroenterol 2012;107:345-360; quiz 361.

3. Gralnek IM, Dumonceau JM, Kuipers EJ, et al. Diagnosis and management of nonvariceal upper gastrointestinal hemorrhage: European 
Society of Gastrointestinal Endoscopy (ESGE) guideline. Endoscopy 2015;47:a1-a46.

4. Rockall TA, Logan RF, Devlin HB, Northfield TC. Risk assessment after acute upper gastrointestinal haemorrhage. Gut 1996;38:316-321.

5. Blatchford O, Murray WR, Blatchford M. A risk score to predict need for treatment for upper-gastrointestinal haemorrhage. Lancet 2000;356:1318-1321.

6. Saltzman JR, Tabak YP, Hyett BH, Sun X, Travis AC, Johannes RS. A simple risk score accurately predicts in-hospital mortality, length of stay, and cost in acute upper GI bleeding. Gastrointest Endosc 2011;74:12151224 .

7. Robertson M, Majumdar A, Boyapati R, et al. Risk stratification in acute upper GI bleeding: comparison of the AIMS65 score with the Glasgow-Blatchford and Rockall scoring systems. Gastrointest Endosc 2016;83:1151-1160.

8. Stanley AJ, Laine L, Dalton HR, et al. Comparison of risk scoring systems for patients presenting with upper gastrointestinal bleeding: international multicentre prospective study. BMJ 2017;356:16432.

9. Park SM, Yeum SC, Kim BW, et al. Comparison of AIMS65 score and other scoring systems for predicting clinical outcomes in Koreans with nonvariceal upper gastrointestinal bleeding. Gut Liver 2016;10:526-531.

10. Gu L, Xu F, Yuan J. Comparison of AIMS65, Glasgow-Blatchford and Rockall scoring approaches in predicting the risk of in-hospital death among emergency hospitalized patients with upper gastrointestinal bleeding: a retrospective observational study in Nanjing, China. BMC Gastroenterol 2018;18:98.

11. Martínez-Cara JG, Jiménez-Rosales R, Úbeda-Muñoz M, de Hierro ML, de Teresa J, Redondo-Cerezo E. Comparison of AIMS65, Glasgow-Blatchford score, and Rockall score in a European series of patients with upper gastrointestinal bleeding: performance when predicting in-hospital and delayed mortality. United European Gastroenterol J 2016;4:371-379.

12. Marmo R, Koch M, Cipolletta L, et al. Predicting mortality in non-variceal upper gastrointestinal bleeders: validation of the Italian PNED score and prospective comparison with the Rockall score. Am J Gastroenterol 2010;105:1284-1291.

13. Jung $\mathrm{SH}, \mathrm{Oh} \mathrm{JH}$, Lee HY, et al. Is the AIMS65 score useful in predicting outcomes in peptic ulcer bleeding? World J Gastroenterol 2014;20:18461851.

14. Romagnuolo J, Barkun AN, Enns R, Armstrong D, Gregor J. Simple clinical predictors may obviate urgent endoscopy in selected patients with nonvariceal upper gastrointestinal tract bleeding. Arch Intern Med 2007;167:265-270.

15. Garcia-Tsao G, Abraldes JG, Berzigotti A, Bosch J. Portal hypertensive bleeding in cirrhosis: risk stratification, diagnosis, and management: 2016 practice guidance by the American Association for the study of liver diseases. Hepatology 2017;65:310-335.

16. de Franchis R. Expanding consensus in portal hypertension: report of the Baveno VI consensus workshop: stratifying risk and individualizing care for portal hypertension. J Hepatol 2015;63:743-752.

17. Villanueva C, Colomo A, Bosch A, et al. Transfusion strategies for acute upper gastrointestinal bleeding. N Engl J Med 2013;368:11-21.

18. Hyett BH, Abougergi MS, Charpentier JP, et al. The AIMS65 score compared with the Glasgow-Blatchford score in predicting outcomes in upper GI bleeding. Gastrointest Endosc 2013;77:551-557.

19. Bryant RV, Kuo P, Williamson K, et al. Performance of the Glasgow-Blatchford score in predicting clinical outcomes and intervention in hospitalized patients with upper GI bleeding. Gastrointest Endosc 2013;78:576-583.

20. Abougergi MS, Charpentier JP, Bethea E, et al. A prospective, multicenter study of the AIMS65 score compared with the Glasgow-Blatchford score in predicting upper gastrointestinal hemorrhage outcomes. J Clin Gastroenterol 2016;50:464-469.

21. Yaka E, Yılmaz S, Doğan N, Pekdemir M. Comparison of the Glasgow-Blatchford and AIMS65 scoring systems for risk stratification in upper gastrointestinal bleeding in the emergency department. Acad Emerg Med 2015;22:22-30.

22. Aquarius M, Smeets FG, Konijn HW, et al. Prospective multicenter validation of the Glasgow Blatchford bleeding score in the management of patients with upper gastrointestinal hemorrhage presenting at an emergency department. Eur J Gastroenterol Hepatol 2015;27:1011-1016.

23. Reed EA, Dalton H, Blatchford O, et al. Is the Glasgow Blatchford score useful in the risk assessment of patients presenting with variceal haemorrhage? Eur J Gastroenterol Hepatol 2014;26:432-437.

24. Choe JW, Kim SY, Hyun JJ, et al. Is the AIMS 65 score useful in prepdicting clinical outcomes in Korean patients with variceal and nonvariceal upper gastrointestinal bleeding? Gut Liver 2017;11:813-820.

25. Thanapirom K, Ridtitid W, Rerknimitr R, et al. Prospective comparison of three risk scoring systems in non-variceal and variceal upper gastrointestinal bleeding. J Gastroenterol Hepatol 2016;31:761-767.

26. Budimir I, Gradišer M, Nikolić M, et al. Glasgow Blatchford, pre-endoscopic Rockall and AIMS65 scores show no difference in predicting rebleeding rate and mortality in variceal bleeding. Scand J Gastroenterol 2016;51:1375-1379.

27. Alexandrino G, Carvalho R, Reis J. Comparison of the AIMS65 score with other risk stratification scores in upper variceal and nonvariceal gastrointestinal bleeding. Gut Liver 2018;12:111-113.

28. Robertson M, Ng J, Abu Shawish W, et al. Risk stratification in acute variceal bleeding: comparison of the AIMS65 score to established upper gastrointestinal bleeding and liver disease severity risk stratification scoring systems in predicting mortality and rebleeding. Dig Endosc 2020;32:761-768

29. Kim BJ, Park MK, Kim SJ, et al. Comparison of scoring systems for the prediction of outcomes in patients with nonvariceal upper gastrointestinal bleeding: a prospective study. Dig Dis Sci 2009;54:2523-2529.

30. Yang HM, Jeon SW, Jung JT, et al. Comparison of scoring systems for nonvariceal upper gastrointestinal bleeding: a multicenter prospective cohort study. J Gastroenterol Hepatol 2016;31:119-125.

31. Nakamura S, Matsumoto T, Sugimori H, Esaki M, Kitazono T, Hashizume M. Emergency endoscopy for acute gastrointestinal bleeding: prognostic value of endoscopic hemostasis and the AIMS65 score in Japanese patients. Dig Endosc 2014;26:369-376. 